\title{
An unusual presentation of an aggressive spindle cell skin cancer
}

Samer A Srour, MBChB, ${ }^{\mathrm{a}, \mathrm{b}}$ Meredith Workman, MD, , Jian T Yang, MD, PhD, ${ }^{\mathrm{d}}$ Sakher A Abdullah, MD, ${ }^{a}$ and Mohamad A Cherry, MDa

${ }^{a}$ Department of Medicine, Section of Hematology/Oncology, ${ }^{b}$ Department of Veteran Affairs Medical Center, ${ }^{\mathrm{c} D e p a r t m e n t}$ of Surgery, Section of Plastic Surgery, and dDepartment of Pathology, The University of Oklahoma Health Sciences Center, Oklahoma City

$\mathrm{S}$ pindle cell squamous cell carcinoma (SCSCC) is a rare subtype of squamous cell carcinoma (SCC) that was first reported around 1900. Its potential for metastasis is uncertain. There has been noted a relationship to previous exposure to radiation therapy with subsequent aggressive presentation. We report an unusual case of a widely metastatic, poorly differentiated cutaneous spindle cell neoplasm with a rapidly progressive clinical course and dismal outcome in several days. This is one of a very few cases in the literature in which a skin cancer recurs with such diffuse metastasis and disastrous outcome.

\section{Case presentation}

A 65-year-old white man presented with multiple rapidly growing facial nodules on the nose, cheek, and forehead as well as a large subcutaneous mass on the back. The patient's past medical history was significant for a SCC of the left shoulder about 4 years before the current presentation that was treated with complete excision followed by adjuvant radiation therapy. In the earlier presentation, the final pathology revealed an invasive high-grade SCC of 7 $\mathrm{cm} \times 3.5 \mathrm{~cm}$. At that time, clear margins were noted in the pathology report and safety margins of $2 \mathrm{~cm}$ were taken circumferentially. The patient did well until his current presentation, when he first noticed a growing mass over his back around 8 weeks before presenting. Two weeks later, he noticed the multiple nodular lesions over his nose, cheek, and forehead (Figure 1) that increased rapidly in size and became necrotic; the nasal lesion was the largest, around 5.0 $\mathrm{cm}$ in maximum dimension.

A review of the patient's systems was significant for weight loss (about $20 \mathrm{lb}$ ), generalized weakness, hemoptysis, shortness of breath on exertion, occa- sional nausea and vomiting, and constipation. On physical examination, the patient looked cachectic. But otherwise there were no significant findings except for the skin and subcutaneous nodular lesions. A skin examination revealed a $5-\mathrm{cm}$ exophytic and necrotic mass on the nasal tip and extending into the septum and to the upper lip. There was also a $2.5-\mathrm{cm}$ lesion on the left lower cheek and a $1.5-\mathrm{cm}$ lesion on the forehead (Figure 1). Examination of the back revealed a subcutaneous mass of $12 \mathrm{~cm} \mathrm{x}$ $10 \mathrm{~cm}$ on the midline of the back, which appeared densely adherent to underlying tissue. The previous excision site on the left shoulder had healed well and there was no evidence of local recurrence.

Multiple punch biopsies of the facial and back lesions were done and the results revealed poorly differentiated spindle cell neoplasm. Immunohistochemistry was negative for pancytokeratin; CK903 and cytokeratin 5 and 6; p63; S-100; HMB-45; pan melanoma; desmin; CD34; and CD117. Tumor cells stained only for vimentin (very nonspecific stain) and no further pathologic characterization of the tumor was possible (Figure 2). Given the patient's history of SCC that had been treated with excision and radiation therapy, the pathologist thought the findings suggested a metastatic spindle cell squamous cell carcinoma.

A positron emission tomography-computed tomography scan revealed unusual diffuse metastatic lesions in the lungs; liver; adrenal glands; pancreas; mesenteric; thoracic; back soft tissues; and the bones and soft tissues of the upper and lower extremities (Figure 3). The patient's condition deteriorated rapidly over 2 weeks during which he was in the hospital, with progressive growth of the aforementioned lesions (Figure $1 \mathrm{~A}$ and $1 \mathrm{~B}$ ), a decline in functional status, and development of respiratory

Accepted for publication October 14, 2013. Correspondence: Samer A Srour, MBChB; samer-srour@ouhsc.edu. Disclosures: The authors have no disclosures. JCSO 2014;12:149-152. (2) 14 Frontline Medical Communications. DOI 10.12788/jicso.0036. 


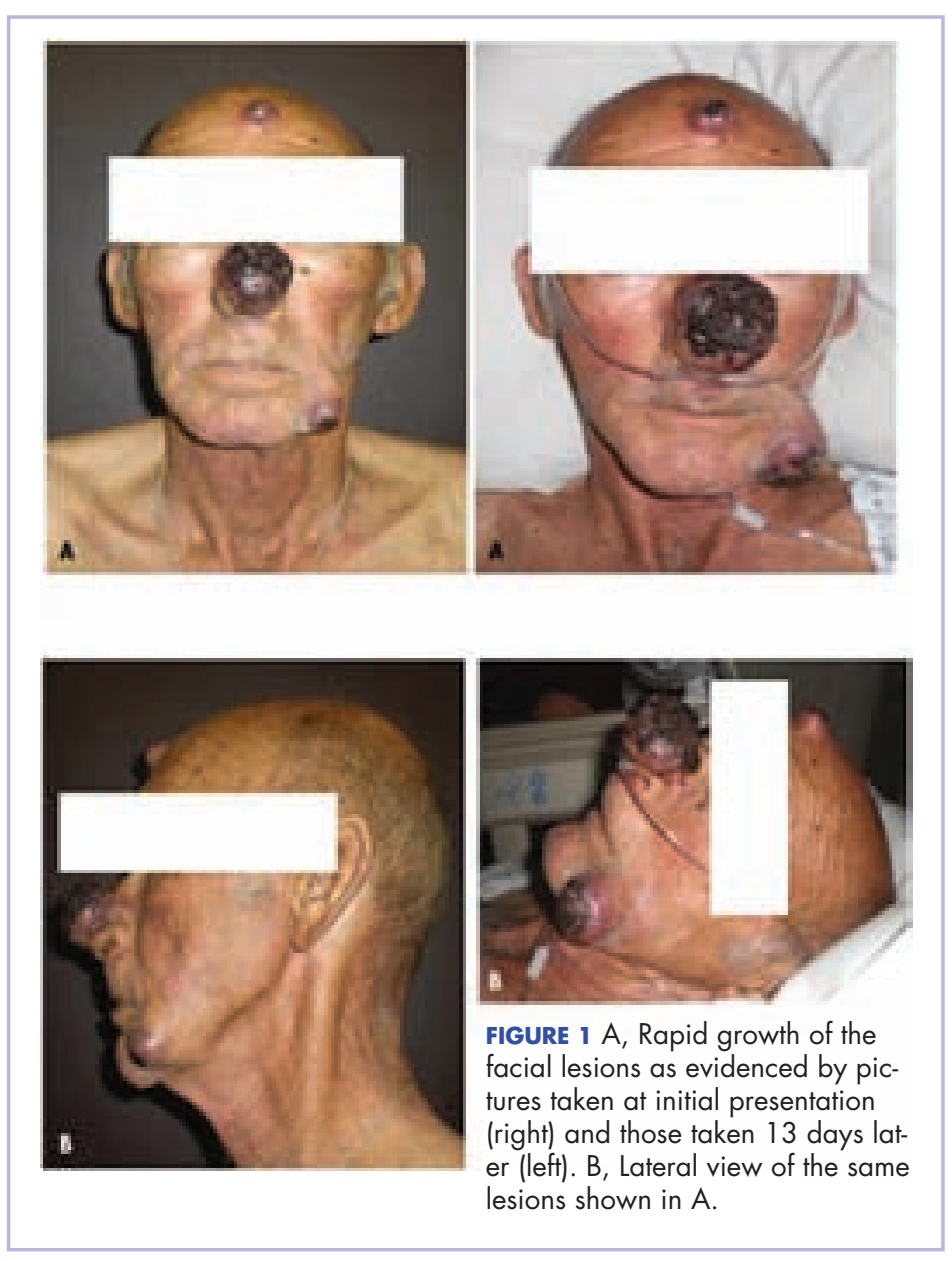

distress. Limited data is available about the best treatment options with such unusual aggressive systematic skin cancer. However, given the patient's poor functional status and the very rapid progression of the disease, the patient elected to proceed with hospice care. He died the day after he was discharged while under care of home hospice.

\section{Discussion}

Nonmelanoma skin cancer (NMSC), which includes both basal cell carcinoma and squamous cell carcinoma, is the most common human malignant neoplasm worldwide. It is more common than all other cancers and has a higher incidence than lung, breast, prostate, and colon cancers combined. ${ }^{1}$ Each year, more than 1 million cases of NMSC are diagnosed in the United States, of which about 20\% are SCC. ${ }^{2}$ The rising incidence of NMSC is likely the result of a combination of factors: growing exposure to sun, more frequent outdoor activities, changes in clothing style, increased longevity, and ozone depletion. ${ }^{3}$ SCC, the second most common human cancer ${ }^{3}$ after basal cell carcinoma, is a neoplasm that arises from malignant proliferation of the keratinocytes of the epidermis with local invasion and metastatic potential.
Although most SCC cases are diagnosed in early stages and have high cure rates (overall 5 -year cure rate is $>90 \%$ ) and an excellent prognosis, ${ }^{4}$ invasive SCC with highrisk features might recur with relatively high potential to metastasize and have a poor treatment outcome. Overall, as per Chartier and Aasi review, rates of metastasis from primary SCC are $2 \%-5 \%$, with about $85 \%$ of metastases occur in regional lymph nodes and the remainder occurring in distant sites..$^{5}$ Ten-year survival rates are less than $20 \%$ for patients with regional lymph node involvement and less than $10 \%$ for patients with distant metastases. ${ }^{6,7}$ Several studies and reviews of SCC have correlated multiple prognostic factors with an increased risk for local recurrence and metastasis. High-risk factors include, but are not limited to, location (higher risk with ear and lip); size ( $>2$ $\mathrm{cm}$ ); depth (> $4 \mathrm{~mm}$ ); histologic differentiation; evidence of perineural involvement; precipitating factors other than ultraviolet light; immunosuppression; presence of acantholysis or desmoplasia; prior treatment modality; rapid growth; and local recurrence of the tumor.,6,8,9 From our review, it seems that depth of the tumor, perineural invasion, poorly differentiated tumors, site, and local recurrence of the tumor have the highest risk of being associated with increased incidence of metastasis (30\%-46\%).

Spindle cell squamous cell carcinoma (SCSCC) is a rare subtype of SCC that was first reported in 1935 but recognized as early as $1900 .{ }^{10}$ The exact incidence of this tumor is unknown, but it is more common in men and is generally reported as an exophytic tumor or an ulcerated mass on the sun-exposed skin of elderly patients. Its gross appearance is frequently indistinguishable from other SCC subtypes. ${ }^{11}$ Among the aforementioned risk factors for SCC and based on brief published reports, it seems that immunosuppression and prior radiation exposure are strongly associated with SCSCC and correlate with aggressive disease and increased metastatic potential compared to de novo SCSCC. ${ }^{10,12-15}$ Although some SCC subtypes such as adenoid and adenosquamous SCC are considered to be markers for increased risk of recurrence and metastasis under National Comprehensive Cancer Network Guidelines, ${ }^{16}$ and SCSCC has been associated with perineural invasion, ${ }^{17} \mathrm{SCSCC}$ is not considered yet a risk factor by itself. ${ }^{16}$

The differential diagnosis of SCSCC includes atypical fibroxanthoma, desmoplastic melanoma, dermatofibrosarcoma protuberans, leiomyosarcoma, and other sarcomas. Diagnosis may be made by morphologic examination, which shows oval malignant cells in a whorl-shaped invasive pattern with a variable degree of keratinization. Mitoses and bizarre pleomorphic giant cells may be frequently seen with other characteristic features. However, in less differentiated cases, it may be difficult to distinguish morphologically from other sarcomas. In these cases, the squamous origin may be confirmed by immunohistochemistry or electron microscopy. Immunohistochemistry is cur- 
rently the primary modality for distinguishing the different subtypes. Atypical fibroxanthoma is characterized by its expression for vimentin, CD68, cathepsin-B, and lack of expression of cytokeratin. Melanomas stain positive for S-100, HMB-45 and pan melanoma stains. SCSCC will typically stain positive for high-molecular weight CK, especially 34betaE12 (CK903) and cytokeratin 5 and 6 , and the p63 marker has been found useful in diagnosis. ${ }^{18-20}$ However, some poorly differentiated SCSCC cases may have complete loss of squamous component and may show loss of cytokeratin expression with aberrant vimentin expression, making the diagnosis even more challenging. ${ }^{12,18,19}$ Electron microscopy can then be used in addition to immunohistochemistry to confirm the diagnosis. The presence of tonofilaments and desmosomes confirms an epithelial origin. ${ }^{21}$

The immunohistochemistry of our case revealed a lack of cytokeratin expression, which is not unexpected given the poorly differentiated appearance on microscopic examination. In fact, vimentin was the only marker that showed positive reactivity, indicating an extensively sarcomatoid tumor. Previous case reports have described how the squamous elements, including cytokeratin expression, may exist only as nests within the tumor, if at all. Taking into consideration that in our case only a few punch biopsies were submitted for pathology and that this represented a fraction of the tumor burden, it is reasonable to believe that squamous elements could be found within other areas of the tumor. Regardless, considering the patient's history of irradiated poorly differentiated cutaneous SCC suggests that his metastatic disease was likely a systemic recurrence of his original skin cancer, which formed the basis for our pathologic diagnosis of SCSCC in his case.

Tumors of mixed epithelial and mesenchymal elements are termed carcinosarcomas. These lesions may arise de novo, ${ }^{13}$ or as degenerative process of preexisting SCC. ${ }^{22}$ The pathogenesis of such tumors is unclear at this point. However, it has been hypothesized that radiation (solar or medical) may induce loss of differentiation of existing carcinoma and conversion to spindle cell morphology. ${ }^{22}$ In our case, the patient originally had squamous cell carcinoma, which was radiated after excision. This is suggestive of the latter pathogenesis.

We hypothesize that the initial treatment to our patient did not cure him. Therefore, the microscopic tumor cells were subjected to radiation therapy after which they might have become less differentiated than the parent tumor cells. Taking into consideration that the original tumor is carcinoma, our case is unique in that it might be a very unusual case of undifferentiated cutaneous spindle cell tumor of likely a carcinoma origin but lacks pankeratin, CK903, and p63 stains, which are usually positive in such types of cancer. This might further explain the aggressive clinical behavior and disastrous outcome.

Controversy exists regarding the potential for SCSCC
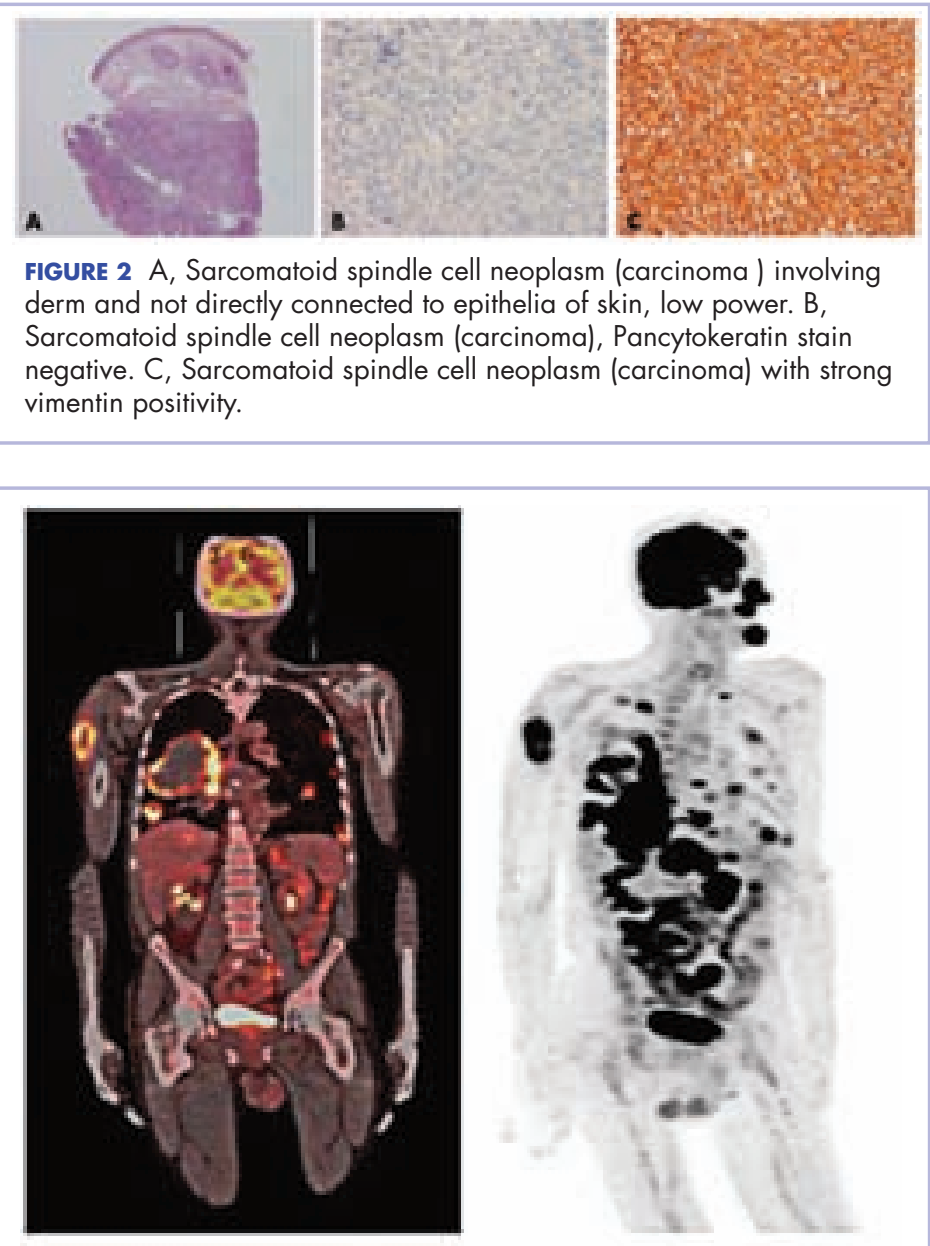

FIGURE 3 A positron emission tomography-computed tomography scan demonstrated widespread metastases involving skin and subcutaneous tissues, bones, muscles, liver, adrenal glands, and lungs.

to metastasize. Though there have been few reports of metastases, and despite the fact such tumors might carry anaplastic features and is frequently associated with perineural invasion, some have reported that they have low frequency of metastasis. ${ }^{11,12,23,24}$ As mentioned above, SCSCC's aggression has been associated with immunosuppression and previous exposure to radiation. It also has shown a connection with the depth of invasion. ${ }^{12}$

Given the rarity of such subtype of SCC, there is no standard of care regarding the best treatment approach for metastatic SCSCC. In general, it is treated as other SCC types. Treatment of nodal disease may involve radiation, lymph node dissection, or both. Treatment of metastatic SCC may include systemic chemotherapy or treatment with biologic response modifiers. The efficacy of these methods has not been established. ${ }^{3}$

\section{Conclusion}

The current case is an extreme example of very aggressive SCSCC, a subtype of SCC, in an immunocompetent 
patient, which probably developed after prior radiation exposure to a microscopic residual SCC. Our case represents one of very few cases in the literature where a skin cancer recurs with such widespread metastases and dismal outcome within couple weeks. This case highlights the challenges pathologists, dermatologists, and oncologists face in trying to better define such rare, undifferentiated tumors and to follow up more frequently with the survivors to ensure an early diagnosis and management of recurrent disease. This should also alert clinicians that, although most skin cancers other than melanoma carry an excellent prognosis, some rare subtypes with certain risk factors are extremely aggressive and harbor dismal prognosis.

\section{References}

1. American Cancer Society. Cancer facts and figures, 2006. http:// www.cancer.org/downloads/STT/CAFF2006PW. September 1, 2010.

2. Jemal A, Siegel R, Ward E, Hao Y, Xu J, Thun MJ. Cancer statistics, 2009. CA Cancer J Clin. 2009;59:225-249.

3. Thomas VD, Aasi SZ, Wilson LD, Leffell DJ. Cancer of the skin. In: DeVita, Hellman, and Rosenberg's Cancer - Principles \& Practice of Oncology. 8 th ed. New York, NY: Lippincott Williams \& Wilkins. 1863-1887.

4. Rowe DE, Carroll RJ, Day CL Jr. Prognostic factors for local recurrence, metastasis, and survival rates in squamous cell carcinoma of the skin, ear, and lip. Implications for treatment modality selection. J Am Acad Dermatol. 1992;26:976-990.

5. Chartier TK, Aasi SZ. Treatment and prognosis of cutaneous squamous cell carcinoma. www.uptodate.com. Last updated January 22,2014. Accessed March 20, 2014.

6. Cherpelis BS, Marcusen C, Lang PG. Prognostic factors for metastasis in squamous cell carcinoma of the skin. Dermatol Surg. 2002;28:268-273.

7. Dinehart SM, Pollack SV. Metastases from squamous cell carcinoma of the skin and lip: an analysis of twenty-seven cases. J Am Acad Dermatol. 1989;21:241-248.

8. Johnson TM, Rowe DE, Nelson BR, Swanson NA. Squamous cell carcinoma of the skin (excluding lip and oral mucosa).J Am Acad Dermatol. 1992;26:467-484.

9. Quaedvlieg PJ, Creytens DH, Epping GG, Peutz-Kootstra CJ, Nieman FH, Thissen MR, Krekels GA. Histopathological character- istics of metastasizing squamous cell carcinoma of the skin and lips. Histopathology. 2006;49:256-264.

10. Martin HE, Stewart FW. Spindle cell epidermoid carcinoma. Am J Cancer. 1935;24:273-297.

11. Petter G, Haustein UF. Histologic subtyping and malignancy assessment of cutaneous squamous cell carcinoma. Dermatol Surg. 2000;26:521-230.

12. Evans HL, Smith JL. Spindle cell squamous carcinomas and sarcoma-like tumors of the skin: a comparative study of 38 cases. Cancer. 1980;45:2687-2697.

13. Smith JL. Spindle cell squamous carcinoma. In: Graham JH, Johnson WC, Helwig EB, eds. Dermal Pathology. Hagerstown, Md: Harper \& Row; 1972:631-635.

14. Harwood CA, Proby CM, McGregor JM, Sheaff MT, Leigh IM, Cerio R. Clinicopathologic features of skin cancer in organ transplant recipients: a retrospective case-control series. J Am Acad Dermatol. 2006;54:290-300.

15. Smith KJ, Hamza S, Skelton H. Histologic features in primary cutaneous squamous cell carcinomas in immunocompromised patients focusing on organ transplant patients. Dermatol Surg. 2004;30:634-641.

16. Miller SJ, Alam M, Andersen J, et al. Basal cell and squamous cell skin cancers. J Natl Compr Canc Netw. 2010;8:836-864.

17. Goepfert H, Dichtel WJ, Medina JE, Lindberg RD, Luna MD. Perineural invasion in squamous cell skin carcinoma of the head and neck. Am J Surg. 1984;148:542-547.

18. Morgan M, Purohit C, Anglin TR. Immunohistochemical distinction of cutaneous spindle cell carcinoma. Am J Dermatopathol. 2008;30:228-232.

19. Folpe AL, Cooper K. Best practices in diagnostic immunohistochemistry: pleomorphic cutaneous spindle cell tumors. Arch Pathol Lab Med. 2007;31:1517-1524.

20. Velazquez EF, Werchniack AE, Granter SR. Desmoplastic/spindle cell squamous cell carcinoma of the skin. A diagnostically challenging tumor mimicking a scar: clinicopathologic and immunohistochemical study of 6 cases. Am J Dermatopathol. 2010;32:333-339.

21. Feldman PS, Barr RJ. Ultrastructure of spindle cell squamous carcinoma. J Cutan Pathol. 1976;3:17-24.

22. Battifora H. Spindle cell carcinoma: ultrastructural evidence of squamous origin and collagen production by the tumor cells. Cancer. 1976;37:2275-2282.

23. Kane CL, Keehn CA, Smithberger E, Glass LF. Histopathology of cutaneous squamous cell carcinoma and its variants. Semin Cutan Med Surg. 2004;23:54-61.

24. Underwood LJ, Montgomery H, Broders AC. Squamous-cell epithelioma that simulates sarcoma. AMA Arch Derm Syphilol. 1951;64:149-158. 